\title{
Actitudes ambientales en la comunidad educativa de la Institución Educativa Rural San Antonio, Sincelejo
}

\section{Environmental attitudes in the educational community of San Antonio, Sincelejo, Sucre}

\section{Berónica Guevara 1.; Pedro Caraballo ${ }^{2}$.}

${ }^{1}$ Maestría en Ciencias Ambientales, Universidad de Sucre. Vero_guevara322@ @otmail.com

${ }^{2}$ Profesor Titular, Universidad de Sucre.pedro.caraballo@unisucre.edu.co

\section{Resumen}

Esta investigación se llevó a cabo en la Comunidad Educativa de la I. E Rural San Antonio, perteneciente al corregimiento de San Antonio, Sincelejo-Sucre. El objetivo fue caracterizar las actitudes ambientales de esa comunidad educativa. Para la caracterización de la Comunidad Educativa, se realizaron observaciones directas en docentes, padres de familia y estudiantes en los que se tenían en cuenta criterios establecidos. Se aplicaron encuestas para evaluar actitudes ambientales en maestros, estudiantes y padres de familia. Se utilizó la Escala de Medición de Actitudes Pro ambientales Infantiles (EMAPI) con una escala de valoración tipo Lickert para estudiantes y padres de familia y en los maestros se aplicó la escala de Enviromental Concern Scale (ECS). Se obtuvo como resultado que un $24 \%$ de los estudiantes, el $23 \%$ de los padres de familia y el $18 \%$ de los maestros muestran actitudes negativas frente alguna serie de acciones como lo es la cacería de animales salvajes y tirar basuras. Luego de aplicar y analizar los resultados se establecieron algunas consideraciones partiendo de lo que la institución se propone en su Proyecto Educativo Institucional y lo manifestado por la comunidad en las encuestas.
Palabras clave: educación ambiental, proyecto educativo institucional.

\begin{abstract}
This research was carried out in the Educational Community of the San Antonio Rural I. E, belonging to the San Antonio village (SincelejoSucre). The objective was to characterize the environmental attitudes of that educational community. For the characterization of the Educational Community, direct observations were made in teachers, parents and students in which established criteria were taken into account. Surveys were applied to evaluate environmental attitudes in teachers, students and parents. The Infant Environmental Attitudes Measurement Scale (EMAPI) was used, with a Lickert rating scale for students and parents and the Enviromental Concern Scale (ECS) scale was applied to the teachers. As a result, $24 \%$ of students, $23 \%$ of parents and $18 \%$ of teachers show negative attitudes towards a several actions such as hunting wild animals and throwing away garbage. After applying and analyzing the results, some considerations were established based on what the institution proposes in its Institutional Educational Project and what the community expressed in the surveys.
\end{abstract}

Keywords: environmental education, institutional educational project 
194

\section{Introducción}

Las identidades personales y colectivas son el resultado de aquello que contamos, a los otros y a nosotros mismos, sobre quiénes somos, integrando al pasado y anticipando el porvenir. Es este el proceso que nos permite dar forma a nuestras vidas y dotarlas de propósito, coherencia, unidad, sentido y significado (Esteban et al, 2008). Las actitudes ambientales que se manifiestan en una comunidad educativa son relevantes. Por ello, la presente investigación se enfoca en evaluar y caracterizar las actitudes ambientales de la comunidad educativa de la I. E Rural San Antonio, permitiendo analizar de manera idónea el comportamiento ambiental de un conjunto de personas que trabajan en forma de sistema en la preparación de estudiantes con un objetivo en común, formar de manera integral a sus educandos. La educación es igualmente fundamental para adquirir conciencia, valores y actitudes, técnicas y comportamientos ecológicos y éticos en consonancia con el desarrollo sustentable, y que favorezcan la participación pública efectiva en el proceso de toma de decisiones (UNESCO, 1992). Es necesario la implementación de este tipo de estudios para consolidar la identidad ambiental y evitar conflictos futuros en la población (Mosquera y Gómez, 2011).

Investigaciones como "cuestiones ambientales que se perciben como problemas" (Aragones y Amérigo, 2006) muestra que la percepción de problemas ambientales está sujeto a una serie de variables como el factor sociodemográfico, intereses políticos, edad, género, inclinación religiosa entre otros factores. La investigación "Escala de actitudes ambientales hacia problemas específicos" (Moreno y Ruiz, 2005) muestra cómo es posible evaluar las actitudes ambientales estableciendo escalas de relación entre una variable ambiental con una actitud específica.

Por lo anterior, se considera importante caracterizar las actitudes ambientales en la Comunidad
Educativa de la I. E Rural San Antonio y establecer que tanta coherencia existe entre lo que el proyecto educativo Institucional busca desde su proyecto de educación ambiental y las actitudes manifestadas por sus miembros. Para la realización de esta investigación se estableció como objetivo caracterizar las actitudes ambientales en la comunidad educativa.

\section{Metodología}

La investigación se llevó a cabo en la I. E Rural San Antonio, ubicada en el corregimiento de San Antonio, en el municipio de Sincelejo- Sucre. Se evaluaron las actitudes ambientales de estudiantes, padres de familia y docentes. En total la comunidad educativa es de 828 personas, tomadas como 3 poblaciones diferentes discriminados de la siguiente forma: 547 estudiantes, 31 docentes y 250 padres de familia aproximadamente. De estas poblaciones se tomó una muestra de docentes, padres de familia y estudiantes. Para el logro de los objetivos específicos:

\subsection{Caracterizar las actitudes ambientales en la comunidad educativa de la I. E Rural San Antonio}

Se hizo una observación directa de las personas que la conforman, dentro y fuera de ella; de modo que se pudo identificar las acciones que normalmente se dan en relación con su ambiente. Los aspectos que se tuvieron en cuenta en la observación son los siguientes: Limpieza del patio y salones de la institución, comportamiento de maestros, directivos y estudiantes durante el descanso y salida de clases, lugar en el que se depositan los desechos generados en la institución, quema de basuras en las calles de la Comunidad indígena San Antonio y Las Huertas, utilización de canecas de basura por parte de maestros y estudiantes, uso adecuado de los baños, ahorro del agua y actividades de reciclaje. La dinámica consistió en observar durante 30 días hábiles el comportamiento de los maestros, directivos y estudiantes durante el desarrollo de la jornada académica $\mathrm{y}$ estos datos se fueron 
registrando en una tabla de frecuencia que indicaba la repetición negativa o positiva de cada uno de los criterios anteriores.

\subsection{Evaluar las actitudes ambientales de la comunidad educativa de la I.E Rural San Antonio}

Para la evaluación de actitudes ambientales en estudiantes y padres de familia, se aplicaron unas encuestas utilizando el instrumento Escala de Medición de Actitudes Pro ambientales Infantiles [EMAPI] con una escala de valoración tipo Lickert en estudiantes y padres familia, el instrumento fue propuesto en el estudio Evaluación psicométrica de un instrumento de medición de actitudes Proambientales en escolares Venezonalos, por (Campos, Pasquiali, Peinanado, 2015.

Para la aplicación del instrumento de evaluación se seleccionó el nivel académico, discriminados así: Nivel de transición 41 estudiantes, la muestra es de 17 estudiantes; en el nivel de Básica Primaria la población es de 231 estudiantes y la muestra seleccionada es de 95 alumnos; en la Básica Secundaria la población es de 207 estudiantes y la muestra es de 85 estudiantes y finalmente el nivel de Media con una población de 68 estudiantes y una muestra de 28; después de definir el número de estudiantes a los que se les aplicó la encuesta, esta cantidad se distribuyó proporcionalmente según el tamaño de cada nivel académico.

Para el análisis de los resultados obtenidos en las encuestas inicialmente se realizó un análisis descriptivo de las variables establecidas en las encuestas de evaluación.

\subsection{Confrontar los resultados de la evaluación de actitudes ambientales en la comunidad educativa con el Proyecto educativo comunitario (PEC)}

Para confrontar estos resultados se determinó cuáles son los temas sustantivos que desde el proyecto Educativo comunitario en su plan de acción del proyecto de educación ambiental se planea formar a estudiantes, maestros y padres de familia de manera positiva en los aspectos evaluados desde las encuestas, así se establece en porcentaje las actitudes positivas y negativas por cada tema.

\subsection{Análisis estadístico}

Se realizó un análisis descriptivo de las variables establecidas en las encuestas de evaluación, en los miembros de la comunidad educativa y se utilizó un cuadro comparativo para establecer las relaciones entre lo esperado desde el proyecto de educación ambiental y la evaluación de las actitudes ambientales.

\section{Resultados}

Se observó una serie conductas negativas diarias y repetidas en maestros y estudiantes frente a los criterios considerados; por ejemplo, se pudo evidenciar que en las áreas de descaso y aulas de la institución permanecen con residuos plásticos. La sala de profesores mantiene residuos de empaques de alimentos, producto de las meriendas de los docentes; así mismo cuentan con unas canecas pero no existe evidencia de su uso adecuado, no se apreció ningún tipo de actividad relacionada con el reciclaje durante el tiempo de observación, ni planeación de este tipo de acciones, adicional a esto, es común ver en las calles principales y alternas de la comunidad indígena de San Antonio y Las Huertas, quema de basuras en las horas de la mañana diariamente.

Para el segundo objetivo se encontró lo siguiente:

Los resultados muestran que el $24 \%$ de los estudiantes encuestados tienen una actitud negativa con relación a aspectos ambientales de la vida en comunidad como se muestra en la Tabla 1, esto es, tirar basuras, cacería de animales silvestres y no les 
196

gusta compartir el uso de vehículos. Por otro lado el $52,04 \%$ de los estudiantes encuestados mostraron actitudes positivas con relación a aspectos ambientales de la vida en comunidad, como se evidencia en la Tabla 2, es decir, cuidar plantas y animales, recoger la basura, les gusta vivir en el campo, están interesados en la energía solar y les preocupa la contaminación del aire.

En los resultados de las encuestas aplicadas en padres de familia, se encontró que el $23 \%$ tiene actitudes negativas frente a los aspectos ambientales relacionados con tirar las basuras, cazar animales silvestres y el uso compartido del carro. Por otro lado, el $31,78 \%$ de los padres dicen tener actitudes positivas frente aspectos relacionados con cuidar animales y plantas, preocuparse por los bosques y la selva tropical, recoger basuras, vivir en el campo, se muestran interesados por la energía solar y se preocupan por la contaminación del aire.

Tabla 1. Porcentaje de las actitudes negativas en padres de familia y estudiantes

\begin{tabular}{|c|c|c|c|c|c|c|}
\hline \multirow{2}{*}{$\begin{array}{l}\text { Preguntas } \\
\text { Negativas }\end{array}$} & \multicolumn{3}{|c|}{ Estudiantes } & \multicolumn{3}{|c|}{ Padres de Familia } \\
\hline & Total si & Muestra & $\%$ & Total si & Muestra & $\%$ \\
\hline $\begin{array}{l}\text { Algunas personas piensan que } \\
\text { las cosas se deben botar } \\
\text { cuando ya no pueden usarse } \\
\text { más. }\end{array}$ & 71 & 245 & 28,98 & 24 & 150 & 16 \\
\hline $\begin{array}{l}\text { A algunas personas no les } \\
\text { gusta hacer comederos } 0 \\
\text { casas para pájaros }\end{array}$ & 63 & 245 & 25,71 & 24 & 150 & 16 \\
\hline $\begin{array}{l}\text { Algunas personas piensan que } \\
\text { los humanos son más } \\
\text { importantes que los animales }\end{array}$ & 56 & 245 & 22,86 & 25 & 150 & 16,67 \\
\hline $\begin{array}{l}\text { Algunas personas no les } \\
\text { preocupa que los animales } \\
\text { desaparezcan (se extingan) }\end{array}$ & 45 & 245 & 18,37 & 77 & 150 & 51,33 \\
\hline $\begin{array}{l}\text { Algunas personas botan las } \\
\text { cosas cuando terminan de } \\
\text { usarlas }\end{array}$ & 59 & 245 & 24,08 & 17 & 150 & 11,33 \\
\hline $\begin{array}{l}\text { Algunas personas no separan } \\
\text { la basura }\end{array}$ & 57 & 245 & 23,27 & 80 & 150 & 53,33 \\
\hline $\begin{array}{l}\text { Algunas personas tocan o } \\
\text { agarran a los animales que } \\
\text { andan libres }\end{array}$ & 76 & 245 & 31,02 & 37 & 150 & 24,67 \\
\hline $\begin{array}{l}\text { A algunas personas no les } \\
\text { gusta compartir carro porque } \\
\text { no les gusta andar apretados }\end{array}$ & 55 & 245 & 22,45 & 25 & 150 & 16,67 \\
\hline $\begin{array}{l}\text { Algunas personas creen que } \\
\text { las personas deben poder vivir } \\
\text { donde quieran }\end{array}$ & 68 & 245 & 27,76 & 30 & 150 & 20 \\
\hline $\begin{array}{l}\text { Algunas personas piensan que } \\
\text { debemos poder cazar todo } \\
\text { tipo de animales salvajes }\end{array}$ & 45 & 245 & 18,37 & 29 & 150 & 19,33 \\
\hline $\begin{array}{l}\text { Algunas personas hacen que } \\
\text { sus padres los leven en carro a } \\
\text { los lugares donde quieren ir. }\end{array}$ & 52 & 245 & 21,22 & 12 & 150 & 8 \\
\hline & & & 24,01 & & & 23,03 \\
\hline
\end{tabular}


Tabla 2. Porcentaje de las actitudes positivas de los estudiantes y padres de familia.

\begin{tabular}{|c|c|c|c|c|c|c|}
\hline \multirow[b]{2}{*}{ Positivas } & \multicolumn{3}{|c|}{ Estudiantes } & \multicolumn{3}{|c|}{ Padres de Familia } \\
\hline & Total si & Muestra & $\%$ & Total si & Muestra & $\%$ \\
\hline $\begin{array}{l}\text { A algunas personas les gusta llevar a } \\
\text { su casa plantas o animalitos que } \\
\text { encuentran. }\end{array}$ & 106 & 245 & 43,27 & 61 & 150 & 40,67 \\
\hline $\begin{array}{l}\text { Algunas personas se preocupan por } \\
\text { los bosques y la selva tropical. }\end{array}$ & 141 & 245 & 57,55 & 25 & 150 & 16,67 \\
\hline $\begin{array}{l}\text { Algunas personas recogen la basura } \\
\text { que los demás echan en nuestros } \\
\text { patios }\end{array}$ & 115 & 245 & 46,94 & 18 & 150 & 12 \\
\hline $\begin{array}{l}\text { A algunas personas les gusta vivir en } \\
\text { el campo donde hay muchas plantas y } \\
\text { animales. }\end{array}$ & 138 & 245 & 56,33 & 26 & 150 & 17,33 \\
\hline $\begin{array}{l}\text { Algunas personas están } \\
\text { entusiasmadas con la energía solar. }\end{array}$ & 124 & 245 & 50,61 & 74 & 150 & 49,33 \\
\hline $\begin{array}{l}\text { Algunas personas se preocupan por la } \\
\text { contaminación del aire. }\end{array}$ & 141 & 245 & 57,55 & 82 & 150 & 54,67 \\
\hline & & & 52,04 & & & 31,78 \\
\hline
\end{tabular}

Finalmente, los docentes muestran un $70,37 \%$ de actitudes positivas cuando se les preguntó por aspectos relacionados con las medidas idóneas para aportar al cuidado de su ambiente, ver Tabla 3. Por otro lado, un 18,10 \% mostró actitudes negativas cuando se les cuestionó por los siguientes aspectos ambientales: No preocuparse por la caza excesiva de animales, consideran que la contaminación no afecta su vida, aunque hay contaminación en aire, suelo y agua piensan que la naturaleza los retornará a su ciclo normal, piensan que los predadores deben ser eliminados y prefieren llevar su carro al trabajo, como se evidencia en la Tabla 4.
Tabla 3. Porcentaje de las actitudes positivas en maestros.

\begin{tabular}{l|l|l|l|}
\hline Positivas & \multicolumn{1}{|c|}{ TOTAL } & $\%$ \\
\hline $\begin{array}{l}\text { El gobierno tiene que introducir duras } \\
\text { medidas para frenar la contaminación, } \\
\text { ya que poca gente la regulará por sí } \\
\text { misma. }\end{array}$ & 26 & 30 & 86,67 \\
\hline $\begin{array}{l}\text { La industria está haciendo los mayores } \\
\text { esfuerzos posibles para desarrollar } \\
\text { tecnología anticontaminante efectiva. }\end{array}$ & 9 & 30 & 30,00 \\
\hline $\begin{array}{l}\text { Estaría dispuesto a hacer sacrificios } \\
\text { personales para reducir el ritmo de } \\
\text { contaminación aunque los resultados } \\
\text { inmediatos no puedan parecer } \\
\text { significativos. }\end{array}$ & 24 & 30 & 80,00 \\
\hline $\begin{array}{l}\text { Debemos prevenir la extinción de } \\
\text { cualquier tipo de animal, incluso si ello } \\
\text { significa sacrificar algunas cosas para } \\
\text { nosotros mismos. }\end{array}$ & 29 & 30 & 96,67 \\
\hline $\begin{array}{l}\text { En la escuela pública deberían ser } \\
\text { impartidos cursos sobre la conservación } \\
\text { de recursos naturales. }\end{array}$ & 30 & 30 & 100,00 \\
\hline
\end{tabular}

Es muy improbable que la

contaminación debida a la producción

$\begin{array}{llll}\text { de energía llegue a ser excesiva porque } & 13 & 30 & 43,33\end{array}$

el gobierno no tiene muy buenas

inspecciones y agencias de control.

El gobierno deberá suministrar a cada

ciudadano una lista de empresas y

$\begin{array}{llll}\text { organizaciones donde se pueda informar } & 25 & 30 & 83,33\end{array}$

de los motivos de queja sobre la

contaminación.

Si pudiera daría tiempo, dinero o ambos para una organización que trabaje para mejorar la calidad del ambiente.

$\begin{array}{lll}21 & 30 & 70,00\end{array}$

Estaría dispuesto a aceptar e

incremento de mis gastos, para

promover el uso prudente de los

$13 \quad 30 \quad 43,33$

recursos naturales. 
Tabla 4. Porcentaje de las actitudes negativas en maestros.

\begin{tabular}{|c|c|c|c|}
\hline Negativas & SI & TOTAL & $\%$ \\
\hline $\begin{array}{l}\text { No deberíamos preocuparnos por matar } \\
\text { demasiados animales de caza, porque a la } \\
\text { larga las cosas se equilibrarán }\end{array}$ & 4 & 30 & 13,33 \\
\hline $\begin{array}{l}\text { La contaminación no afecta } \\
\text { personalmente a mi vida. }\end{array}$ & 5 & 30 & 16,67 \\
\hline $\begin{array}{l}\text { Los beneficios de los productos de } \\
\text { consumo modernos son más importantes } \\
\text { que la contaminación que resulta de su } \\
\text { producción y uso. }\end{array}$ & 2 & 30 & 6,67 \\
\hline $\begin{array}{l}\text { Aunque hay contaminación continua en } \\
\text { lagos, ríos y aire, los procesos de } \\
\text { purificación de la naturaleza pronto los } \\
\text { retornan a lo normal. }\end{array}$ & 6 & 30 & 20,00 \\
\hline $\begin{array}{l}\text { Los predadores tales como los halcones, } \\
\text { cuervos, zorros y lobos que viven de las } \\
\text { cosechas de grano y aves de corral de los } \\
\text { granjeros deberían ser eliminados. }\end{array}$ & 3 & 30 & 10,00 \\
\hline $\begin{array}{l}\text { La actividad corriente de las } \\
\text { organizaciones anticontaminación está } \\
\text { realmente más interesada en romper con } \\
\text { la sociedad que en luchar contra la } \\
\text { contaminación. }\end{array}$ & 10 & 30 & 33,33 \\
\hline $\begin{array}{l}\text { Incluso si el transporte público fuera más } \\
\text { eficiente de lo que es, yo preferiría llevar } \\
\text { mi carro al trabajo. }\end{array}$ & 8 & 30 & 26,67 \\
\hline & & & 18,10 \\
\hline
\end{tabular}

\section{Conclusiones}

En la comunidad educativa de la Institución educativa no existe coherencia entre su plan de acción desde el proyecto de educación ambiental, la evaluación de sus actitudes ambientales y la forma cómo ellos se consideran frente algunas criterios ambientales, es decir, se evalúan como personas con identidad ambiental positiva, pero su actitud ambiental refleja otra perspectiva de la realidad, concluyendo así la necesidad de intervenir pedagógicamente esta comunidad, planteando reformas estructurales en su Proyecto de educación ambiental de manera que sea más coherente, significativo y pertinente con las necesidades de la comunidad educativa

\section{Agradecimientos}

A la Comunidad Educativa de la I. E Rural San Antonio

\section{Referencias Bibliografícas}

Martínez Ponce, I. N. (2015). Identidad ambiental: La construcción de un concepto a partir del análisis de la plataforma Pro-Río. http://hdl.handle.net/10045/53459

Aragones, J. I., \& Amerigo, M. (1991). Un estudio empírico sobre las actitudes ambientales. Revista de Psicología Social, 6(2), 223-240. https://dialnet.unirioja.es/descarga/articulo /111760.pd

Campos, M. L., Pasquali, C., \& Peinado, S. (2015). Evaluación psicométrica de un instrumento de medición de actitudes pro ambientales en escolares venezolanos. Paradigma, 29(2), 135-156.

revistas.upel.edu.ve/index.php/paradigma/ article/view/1935.

Aragonés, J. I., Sevillano, V., Cortés, B., \& Amérigo, M. (2006). Cuestiones ambientales 
199

que se perciben como problemas. Medio ambiente y comportamiento humano, 7(2), 119. https://mach.webs.ull.es/PDFS/Vol7 2/Vol 72 a.pdf

Moreno, M., Corraliza, J. A., \& Ruiz, J. P. (2005). Escala de actitudes ambientales hacia problemas específicos. Psicothema, 17(3), 502-508.

www.psicothema.com/psicothema.asp?id $=3136$

Mosquera Téllez, J., \& Gómez Carvajal, E. R. (2011). La gestión del riesgo-de la incertidumbre a la adaptibilidad. Bistua: Revista de la Facultad de Ciencias Básicas, 9(1). www.redalyc.org/service/redalyc/...gestión ...riesgo...incertidumbre....adaptibilidad/1

Berónica Guevara. Licenciada en Educación con énfasis en Ciencias Naturales y Educación ambiental. Candidata a Magíster en Ciencias ambientales en la Universidad de Sucre. Docente de aula en la Alcaldía del municipio de Sincelejo. _ORCID Id 0000-0001-6202338X.

Pedro Caraballo. Biólogo Marino, MSc y Doctor en Ecología.Profesor en la Universidad de Sucre; investiga la identidad ambiental como elemento de conservación de la naturaleza. ORCID Id 0000-0001-9050-7080.

*Para citar este artículo: Guevara B.; Caraballo P. Environmental attitudes in the educational community of San Antonio, Sincelejo, Sucre . Revista Bistua.2018.16(1):193-199

+ Autor para el envió de correspondencia y la solicitud de las separatas: Caraballo P.I Universidad de Sucre.pedro.caraballo@unisucre.edu.co
Recibido: Julio 14 de 2017

Aceptado: Febrero 05 de 2018 